\title{
UN NOMBRE EN UN POEMA DE KAVAFIS'
}

\section{Miguel Castillo Didier Universidad de Chile. Chile.}

Resumen: En este artículo se expone el problema que se ha planteado a muchos

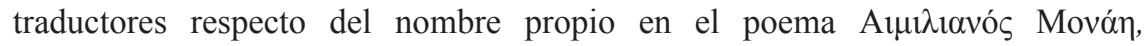
$\mathrm{A} \lambda \varepsilon \xi \alpha v \delta \rho \varepsilon v ́ \varsigma, 628-655 \mathrm{M}$. X. Se presentan múltiples traducciones en las que se ha incurrido en un error en la traducción del nombre del personaje. Se plantea la tesis de que la solución del problema la dio el propio poeta en carta a su hermano John, quien le había pedido que le diera la transcripción en caracteres latinos del nombre. En 2003, Manolis Savidis publicó las versiones de 63 poemas hechas por John. La del poema mencionado deja en claro el sentido que el poeta daba al nombre del personaje.

Palabras claves: C. P. Kavafis - John Kavafis - Emiliano Monai - M. Savidis.

\section{A NAME IN A CAVAFY'S POEM}

Abstract: This article presents the problem that has been posed to many transla-

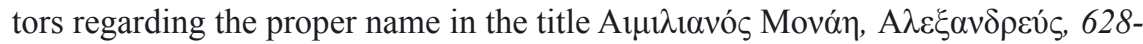
$655 \mathrm{M}$. X. Multiple translations are presented in which an error has been made in the translation of the character's name. The thesis is raised that the solution of the problem was given by the poet himself in a letter to his brother John, who had asked him to give the transcript in Latin characters of the name. In 2003, Manolis Savidis published the translations of 63 poems made by John. The translation of the poem mentioned make clear the meaning that the poet gave to the character' name.

Keywords: C. P. Cavafy - John Cavafy - Aimilian Monai - M. Savidis.

Recibido: 15.10.2019 - Aceptado: 31.03.2020

Correspondencia: Miguel Castillo Didier

Email: micastilgriego@gmail.com

Profesor titular de la Universidad de Chile. Casilla 435-3, Santiago, Chile.

1 Este trabajo tiene su origen en el proyecto "Kavafis: Cartas al joven Kavafis", registrado en la Comisión de Investigación y Publicaciones de la Facultad de Filosofía de la Universidad de Chile, año 2020. 


\section{Los epistolarios perdidos}

e los tres grandes epistolarios de Constantino correspon-
dientes a los tres años en que parte de la familia vivió en
Constantinopla (1882-1885), es de lamentar especialísimamente la pérdida de las cartas que envió Kavafis a su hermano John, dada la estrecha colaboración intelectual y literaria que hubo entre ellos en los años juveniles, como se desprende de las misivas de John. Sabemos que, desafortunadamente, esas cartas fueron destruidas por su sobrina Jariclia Kavafi (después Jariclia Valieri), quien vivía con su tío a la fecha de su muerte, en 1923. La noticia que da Savidis es bien clara:

"Hago notar que, de acuerdo con el testimonio oral de Jariclia Valieri, las cartas de Constantino a John, fueron destruidas por ella después de la muerte de John por expreso deseo de éste"2.

No es fácil imaginar la causa de la idea de John de destruir esas cartas. Se ha insinuado que las relaciones entre los hermanos se habrían enfriado en los últimos años. Al menos hasta la muerte de Pablo, en Francia, en 1920, las relaciones entre ellos parecen conservar el afecto que siempre se demostraron. A la luz de lo que aquí expondremos, resulta posible una explicación para la decisión de John de ordenar quemar las cartas de su hermano.

El especial afecto que tenía John por Constantino se muestra no pocas veces a su correspondencia. No pocas veces en las cartas de los años 1882-1885, contienen expresiones como "sabio Constantino", "mi sabio Mentor", "mi Mentor", etc 3 .

Una carta de 24 de enero de 1920, cuando Constantino tenía 57 años y John tenía 59 años (morirá de 61), éste expresa ese cariño y admiración en pocas palabras. Antes se refiere, con tristeza a su propia vida que ve fracasada:

2 Y. P. Savidis: Mıкрá Kaßapıкá A Pequeños estudios kavafianos I., p. 52, nota 29. Vanyelis Karayanis se refiere al destino de otros papeles personales de John: "Cuando murió John, por orden anterior de éste, Valieri [Jariclia Kavafi], ella misma, puso todos sus papeles personales, etc., bajo su almohada, en la urna, con excepción de su correspondencia con Kiti Ionidi (hija de Ioanis Georgíu)". V. Karayanis (1983), p. 149, nota 14. 
"Mi alma me anuncia que mi vida está terminada y acabada y lo que me descorazona es el magro resultado que puedo mostrar por los muchos años que he vivido... Pero no quiero entristecerte. Tú eres mi hermano, y eso no es poco; estoy orgulloso de ti y de tu obra, y me esfuerzo lo más que puedo por hacer conocido el valor de esa obra entre mi pequeño círculo de relaciones angloparlantes"4.

El 9 de mayo de 1919, The Athenaeum publicó en Londres, en la página 311, Sección "Correspondence", una carta firmada por E. M. Forster y George Valasópulos 5 , en el texto de la cual se presentan los poemas Mar de la Mañana y Reyes alejandrinos. Los firmantes llaman la atención del Director de la revista acerca de una errata en "nuestro artículo" sobre la poesía de C. P. Kavafis, publicado en el número del 25 de abril. Dicho artículo, conocido tradicionalmente como el primer "ensayo" de Forster sobre la poesía de Kavafis, reprodujo tres poemas: Mar de la mañana, En el mes de Athyr, Reyes alejandrinos, además de breves fragmentos de Itaca y de La ciudad. El artículo fue reproducido luego por la Egyptian Gazette de Alejandría 6 .

John se tiene que haber enterado, por lo tanto, de que el autor no utilizaba traducciones de las que él, a través de años había hecho de los poemas de su hermano. Acaso haya pensado que Forster no se las pidió a Constantino, sino a su amigo Valasópulos [Valasópulos había sido su condiscípulo en el King's College]. Pero también puede haber pensado que su hermano fue consultado y aprobó el que se incluyeran versiones de Valasópulo. Esta última idea tenía que producirle un profundo dolor. Y tal idea correspondía a la realidad, como lo comprobamos por la carta de Kavafis a Forster, de $1^{\circ}$ de octubre 1919, en la que leemos: "Le pedí a Valasópulos que enviara a usted más traducciones de mis poemas"7.

$4 \quad$ Carta 74 de John.

5 Esta carta se reproduce en fotografía, en la lámina 10 de The ForsterCavafy Letters...

6 En Pharos and Pharilllon, Forster reproduce en 1923 el ensayo y figuran en el texto traducciones de los poemas Mar de la mañana, En el mes de Athyr y Reyes alejandrinos y fragmentos de La ciudad y de Itaca.

The Forster-Cavafy Letters, p. 44. 
Peter Jeffreys escribe en una nota: "Muy curiosamente, John sometió sus propias traducciones de poemas de su hermano a The Athenaeum en 1919. Una carta del editor (John Middleton Murry) las rechazó, dando como razón el que los mismos poemas habían ya aparecido en The Athenaeum en versiones de Valasópulo" ". A continuación Jefreys añade: "Este extraño episodio provoca la seductora pregunta de si Kavafis supo o alentó el esfuerzo del su hermano por competir con Valasópulos".

No sabemos si Kavafis supo del envío de los poemas traducidos por John a la revista, pero es claro que no alentó el esfuerzo de su hermano. Lo más probable es que no se haya hablado más sobre las traducciones entre los hermanos.

Nosotros pensamos que para John era natural que sus traducciones, hechas con gran cariño, consultadas con su hermano, se publicaran alguna vez. El inglés, idioma que él dominaba perfectamente y que lo había utilizado profesional y literariamente toda su vida, era la lengua que daría salida al mundo a la creación de su hermano. Ese era su "acariciado proyecto". El enterarse de que se habían publicado versiones de otra persona tiene que haber producido un gran dolor a John. Tomó la iniciativa de ofrecer a la revista sus versiones y ellas podrían haber sido aceptadas si la dirección de la revista hubiera tenido un criterio amplio. La publicación podría haber dado lugar a una interesante actividad de comparación de las versiones.

Es posible que la carta citada más arriba, de 24 de enero de 1920, en la que John habla de sus esfuerzos por hacer conocida la obra de su hermano en la comunidad angloparlante de Alejandría, haya sido una manera de "reclamar", suavemente, por lo que consideraba una gran injusticia. Se habría sentido en cierto modo traicionado por su hermano, a quien desde muy joven había pronosticado un futuro reconocimiento de su poesía y que había trabajado por la difusión de ella en los círculos anglófonos de Alejandría en los cuales él siempre se movió. Es posible que esto haya provocado su orden de hacer desaparecer las cartas de Constantino.

Sabemos que John juntaba y guardaba cuidadosamente las cartas que recibía de su hermano, durante los tres años en que éste permaneció en Constantinopla, entre 1882 y 1885 . Escribía, en efecto, el 2 de octubre de 1882:

8 The Forster-Cavafy Letters..., nota 64, p. 29. Jeffreys no documenta su afirmación. 
"No estoy seguro de que mis cartas sean dignas de ser guardadas y nadie sino tú encontrarás algún interés en la lectura atenta de las mismas. Las tuyas yo las guardo religiosamente en un cuaderno de tapas de tela rotulado "Cartas de Constantino Fotiadès Cavafy", depositado seguro con llave, en un cajón de mi escritorio en mi oficina. Las saco de cuando en cuando, y es decir la pura verdad de que las leo de nuevo con tanto interés como cuando recién las recibí'".

Y en otra carta, de 14 de mayo de 1884, leemos la siguiente afirmación:

“Tus cartas son siempre atesoradas por mí y se mantienen en un lugar seguro, con algunos otros de mis objetos de valor"10.

Por lo tanto, si no hubieran sido destruidas, a su muerte habrían quedado en un ordenado archivo, tal como permanecieron las que él enviaba a su hermano Constantino. Sólo aquel gran dolor pudo decidirlo a disponer la destrucción de esos textos que tanto había apreciado y que había guardado con tanto cuidado.

Jariclia Kavafi, después condesa Jariclia Valieri, sobrina muy querida del poeta, hija de Arístides Kavafis, en un capítulo de sus Memorias, titulado "Mi tío Kostís", escribe "Hasta entre sus hermanos, Kostís no encontraba comprensión, salvo en John, que era un valioso poeta anglófono y que había traducido bastantes poemas de su hermano"11.

Equivocadamente, Jariclia Valieri, en una entrevista dada a Vanyelis Karayanis el 16 de agosto de 1968, afirma: "Desde 1919 hasta 1923, en que vivimos con John, no hizo ninguna traducción de Constantino"12. Tenemos una prueba, prueba muy importante como veremos, de que en 1920, John estaba traduciendo poemas de su hermano.

En efecto, en carta del 19 de enero de ese año, le pide a Constantino "Escríbeme, por favor, en caracteres latinos el nombre propio A $\mu \iota \imath \imath \alpha$ vó Movón”. En la respuesta, que no tenemos pues fue quemada, Kavafis le dio

$9 \quad$ Carta 5 de John.

10 Carta 45 de John.

11 Valieri, J. (1983), p. 93.

12 Karayanis, V. (1983), p. 149. 
la transcripción, pensando naturalmente en que se trataba de una traducción al inglés, como todas las que había hecho John.

La transcripción que el poeta dio a su hermano la conocemos desde el año 2003, cuando Manolis Savidis editó las 63 traducciones de John. Esta es la transcripción contenida en el título del poema y en el poema mismo: "Aemilian, the son of Monaes".

Según informa Manolis Savidis en la "Introducción" a su edición de las traducciones de John, "los poemas se publican aquí en el orden en que aparecen en los dos últimos cuadernos [de siete que existen en el archivo]". En esa ordenación, el título del poema es Aemilian, the son of Monaes, of Alexandria, 628-655 A.D. y es el sexagésimo primer texto. Le siguen Alexandrian Kings y The grave of Lysias the Grammarian. Es pues de suponer que estos son los tres últimos poemas traducidos por John. En enero de 1920, John estaba traduciendo el poema sobre Aıııııvós Movół. Alguna duda debe haber tenido y por eso le pidió a su hermano que le diera la transcripción en caracteres latinos del nombre del personaje. El hecho de que le consultara era también acaso una manera de recordarle que él seguía en el trabajo de traducir poemas kavafianos.

La publicación de las traducciones de John, permite corregir las tra-

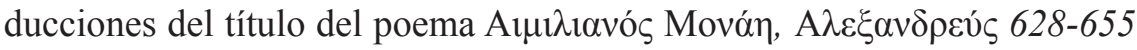
M. X., la cuales, con algunas variaciones de detalle, han caído en el mismo error. En efecto, en una inmensa cantidad de traducciones, incluida la nuestra, o se transcribe simplemente la palabra Movó $\eta$ por Monai o por Monaes o por Monae.

Nosotros lo tradujimos Emiliano Monai. El profesor Pedro Bádenas de la Peña traduce Emiliano Monaes. Edmund Keeley y Philip Sherrard traducen Aimilian Monai.

A continuación, entregamos una lista de veintiún traductores a los siguientes idiomas: castellano, inglés, francés, rumano, alemán, holandés, italiano y portugués, y la versión que ha dado cada uno al nombre de Emiliano. 


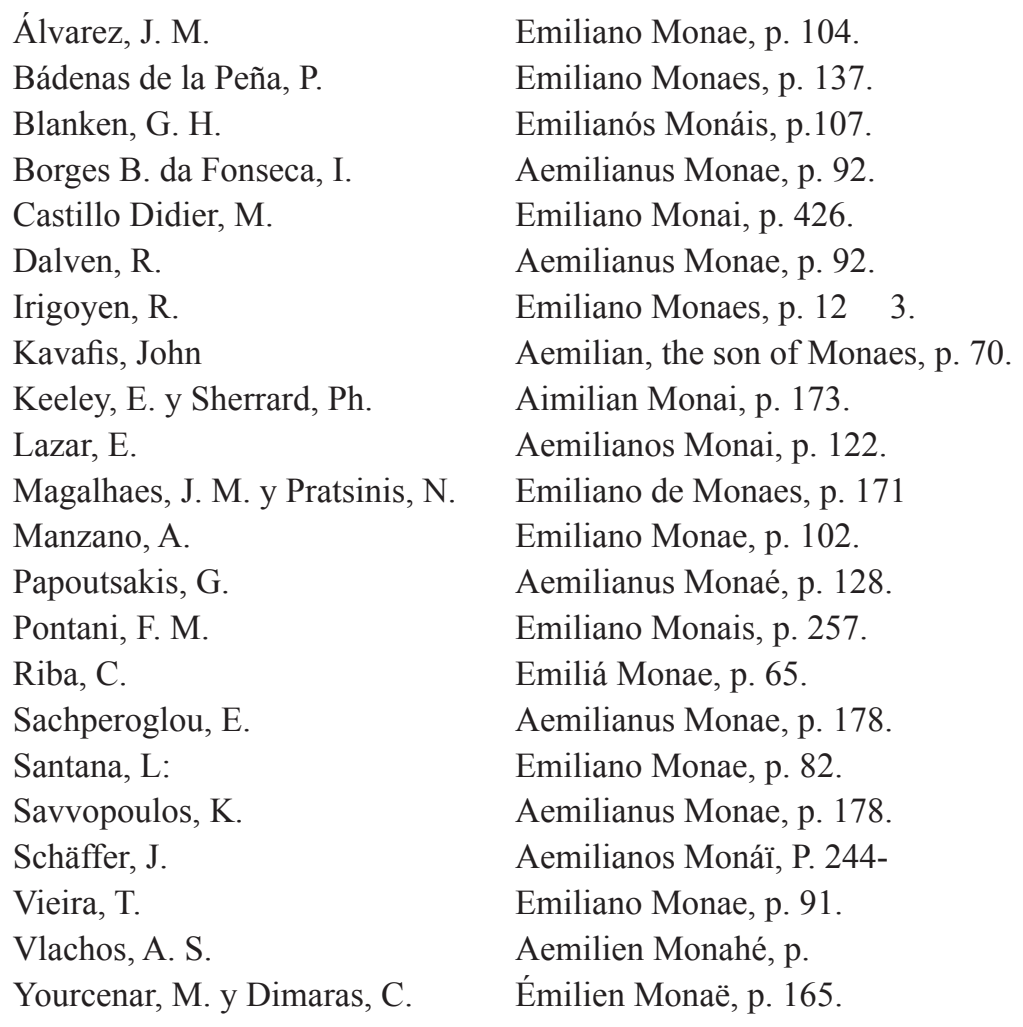

Como lo habíamos anotado más arriba y como puede verse leyendo esta lista, todos los traductores escriben o Monai o Monae o Monaes, con pequeñas variantes de escrituras: Monaé, Monáis, Monaï, Monaë.

Joaquin Manuel Magalhaes y Nikos Pratsinis, en cambio, escriben Emiliano de Monaes (. P 171), al parecer traduciendo literalmente el antiguo genitivo de procedencia como "de" y no como "hijo de".

Entre los 46 poemas traducidos por Valasópulos y que se publican en el volumen The Forster-Cavafy Letters, no figura el de Emiliano, por lo cual el único testimonio de la forma en que el poeta quiso dar a la traducción de Movón está en la versión de John ${ }^{13}$.

13 The Forster.Cavafy Letters, pp. 159-184. Del poema La tumba de Lanes hay dos versiones. Frente a la página 158, en imagen sin numeración, se publica una traducción de La tumba de Lanes, mecanografiada por Valasópulos, con numerosas correcciones manuscritas de Kavafis. Esto muestra que posiblemente 
Ahora queda claro que la palabra Movón es genitivo de Movóns.

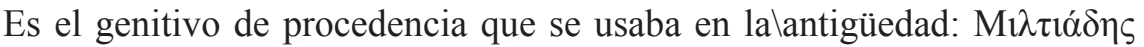

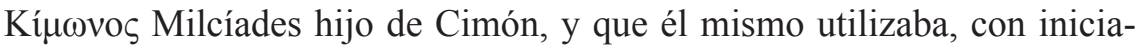
les, en su firma Constantino P[etru] Kavafis: Constantino [hijo de Pedro] Kavafis. El nombre de su padre era, como sabemos, Petros Ioanis, Pedro Juan Kavafis.

hubo una intensa intervención del poeta en la elaboración de las traducciones de Valasópulos. Del mismo modo, Kavafis podría haber corregido versiones de su hermano si hubiera querido presentarlas. 
AIMILIANOS MONAH ALEXANDREUS, 628-655 M.C.

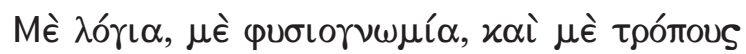

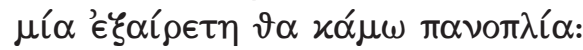

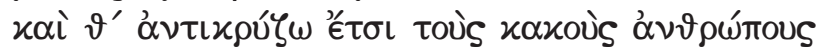

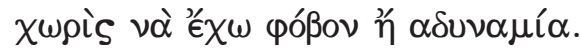

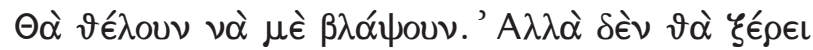

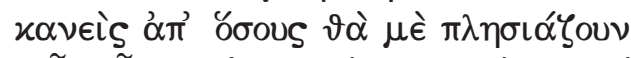

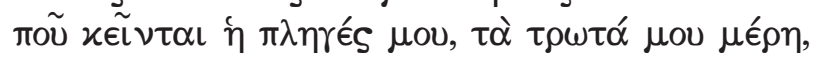

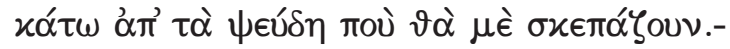

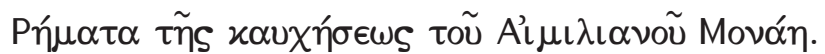

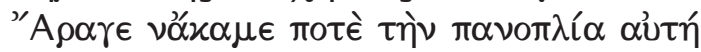

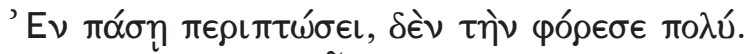

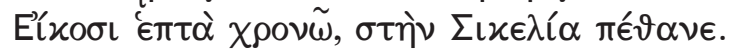

\section{Emiliano hijo de Monais, alejandrino, 628-655 d. C.}

Con palabras, con apariencia y con maneras una excelente coraza he de forjarme:

y he de enfrentar así a los hombres malos sin tener miedo o debilidad.

Querrán dañarme. Pero no ha de saber ninguno de cuantos se me acerquen dónde están mis heridas, mis puntos vulnerables, bajo las falsedades que me recubrirán.-

Palabras de jactancia de Emiliano hijo de Monais. ¿Llegó alguna vez a forjarse tal coraza?

En todo caso, no la llevó por mucho tiempo.

De veintisiete años, en Sicilia murió. 


\section{AEMILIAN, THE SON OF MONAES, OF AI.EXANDRIA, 628-655 A.D.}

My words, and my appearance, and my way, shall fashion me an armour excellent; and so, being from every fear and every weak ness wholly free, I shall confront the men who are on evil ends intent.

They shall desire to injure me. But who shall say of all of those approaching me, that I have wounds? or who of them shall know which are the parts where I am vulnerable, beneath my panoply of lies unfathomable?

With boastful spirit, and in words resembling these, ran thought and speech of young Aemilian, the son of Mónaes: Did he, one wonders, ever have that armour made? Howbeit, he did not wear it long, for he was laid at rest, in Sicily, at the age of twenty-seven. 


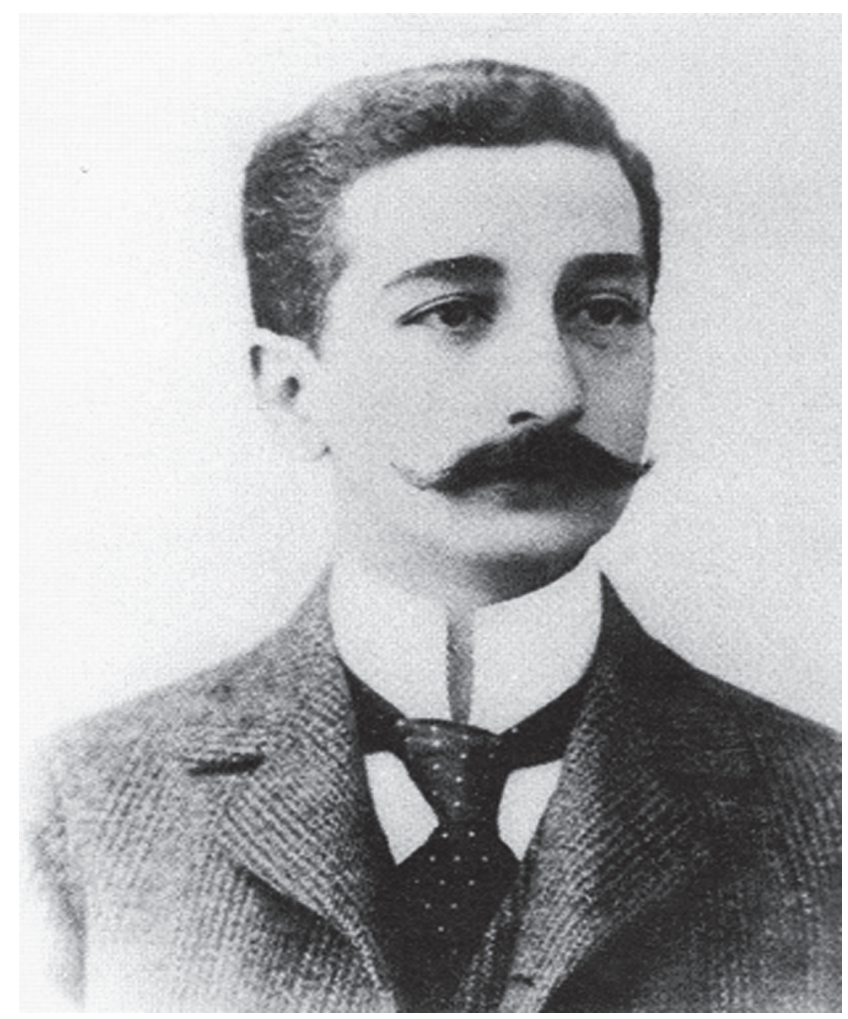

John Kavafis (Ioanis Constantinos Kavafis)

1861-1923 


\section{К. П. КАВАФН}

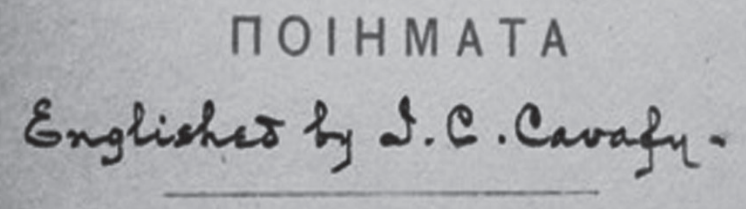

A $A E \equiv A N \triangle P E I A$

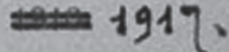

Uno de los cuadernos de traducciones de John, que utiliza una de las portadas que ponía Kavafis a sus colecciones de "feuilles volantes". 


\section{C.P. Cavafy}

sixty-three pocms translated by

J.C. Cavafy

ven whem.

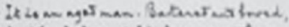

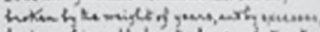

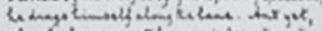

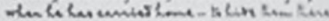

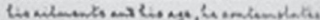

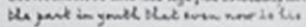

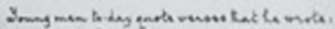

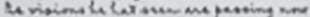

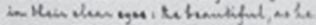

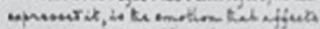

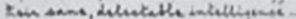

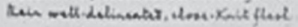

Edición de los sesentitrés poemas de Kavafis traducidos por John, publicada por Manolis Savidis en Atenas el año 2003. 


\section{Los epistolarios conservados}

Son tres: Cartas a Forster, Cartas a Vaianos y Cartas a Aleko Sengópulos. A los dos primeros dedicamos el libro Kavafis: cartas a Forster y a Vaianos. En él utilizamos y comentamos el trabajo del profesor Dimitris Daskalópulos dedicado a "C. P. Kavafis como escritor de cartas". De los epistolarios perdidos, estudiamos el de Ioanis Constandinos en el libro Cartas al joven Kavafis.

\section{REFERENCIAS BIBIOGRÁFICAS}

Se registran primeramente las traducciones por orden alfabético de los nombres de los traductores.

ÁlvareZ, J. M. (1976) Konstantino Kavafis Poesías Completas. Madrid: I. Peralta Ediciones San Fermín.

BÁDENAS DE LA PEÑA, P. (1997) C. P. Kavafis Poesía Completa. Introducción y notas Pedro Bádenas de la Peña.Madrid: Alianza Editorial.

BLANKEN, G. H. (1962) K. P. Kavafis 100 Gedichten. Uit net Nieuwgrieeks

Vertaald door G. H. Blanken. Amsterdan: Moussault's Uitgeverig N.V.

BORGES B. DA FONSECA, I. (2006) Constantinos Kavafis Poemas. Introduçāo

e notas Isis B. da Fonseca. Sao Paulo: Odysseus Editora Ltda.

CASTILLO DIDIER, M. (2007) Kavafis íntegro. Santiago: Tajamar EditoresCentro de Estudios Griegos Universidad de Chile.

DALVEN, R. (1966) The Complete Poems of Cavafy Translated by Rae Dalven with an Introduction by W. H. Auden. London: The Hogarth Press Ltd.

IRIGOYEN, R. (1994) C. P. Cavafis Poemas. Traducción y prólogo R. Irigoyen. Barcelona: Seix Barral.

KAVAFIS, J. (2003) C. P. Cavafy Sixty three poems translated by J. C. Cavafy. With an Introduction by Manuel Savidis. Atenas: Íkaros.

LAZAR, E. (1993) Kavafis Opera poética. Cuvant inainte de Dimitris Daskalopulos Traducere, prezentari, note si comentarii de Elena Lazar. Bucuresti: Editura Omonia.

MAGALHAES, J. M. Y PRATSINIS, N. (2005) Konstandinos Kavafis Os Poemas. Traduçāo do grego, prefacio e notas J. M. Magalhaes e N. Pratsinis. Sta. Maria de Feira: Relógio D’Água Editores.

MANZANO, A. (1984) Kavafis Obra Escogida. Barcelona: Editorial Teorema S. A.

PAPOUTSAKIS, G. (1958) C. P. Cavafy Poèmes. Traduits par G. P. Préface de André Mirambel. París : Les Belles Lettres. 
PONTANI, F. M. (1961) Costantino Kavafis Poesie A cura di Filippo Maria Pontani. Roma: Arnoldo Mondadori Editore.

RIBA, C. (1962) Poemes de Kavafis Selecció i traducció Carles Riba.II. Ilustracions de J. Subirachs. Nota preliminar de Joan Triadú. Barcelona: Editorial Teide.

SANTANA, L. (1973) Constantino Cavafis 75 Poemas. Versión, prólogo y notas de L. Santana. Madrid: Visor.

SAVVOPOULOS, K. (2013) A Historial Guide to Cavafy's Alexandria (331 BCE - 641 CE) Translation of poems by Evangelos Sachperoglou. Alejandría : Bibliotheca Alexandrina.

SCHÄFFER, J. (2003) Konstantin Kavafis Das Hauptwerk Gedichte griecisch und deutsch übersetzt und commentiert von Jörg Schäffer mit Bildnismünzen ausgewählt und kommentiert von Peter Robert Franke. Heidelberg : Universitätsverlag Winter.

VIEIRA, T. (2007) Konstantinos Kaváfis 60 Poemas. Seleçāo e Traduçāo T. Vieira. Cotia Cotia: Atelié Editorial.

YOURCENAR, M. (1958) Présentation Critique de Constantin Cavafy 18631933.Suivi d'une traduction intégrale de ses poèmes par Marguerite Yourcenar et Constantin Dimaras. París : Gallimard.

\section{Algunos estudios}

CASTILLO DIDIER, M. (2019) Kavafis: Cartas a Forster y a Vaianos. Santiago: Centro de Estudios Griegos.

--- (2020) Cartas al joven Kavafis Las cartas de John. Santiago: Centro de Estudios Griegos.

--- (2008) "Kavafis: introducción a tres epistolarios. Los epistolarios perdidos". Byzantion Nea Hellás № 27 (Santiago).

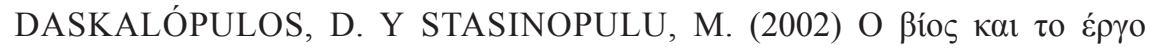

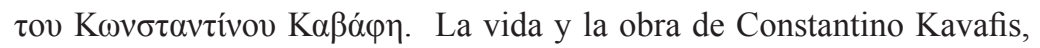
Atenas: Metejmio.

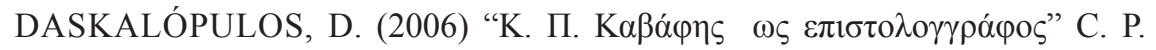

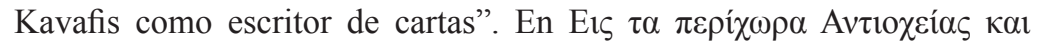

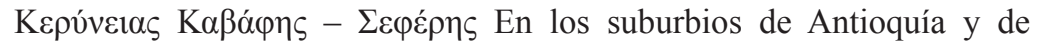
Kerinia Kavafis - Seferis. Atenas: Íkaros. 


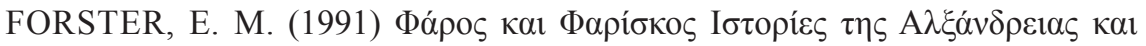

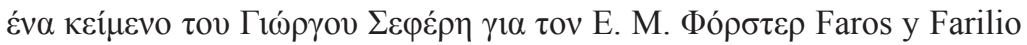
Historias de Alejandría y un texto de Yorgos Seferis sobre E. M. Forster. Traducción Any Spirakou. Atenas: Ediciones Alexandria.

JEFFREYS, P. (2009) "Introduction" a The Forster-Cavafy Letters Friends at a Slight Angle. Edited and Annotated by Peter Jeffreys. El Cairo: The American University in Cairo Press.

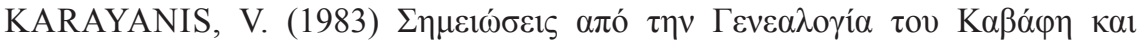

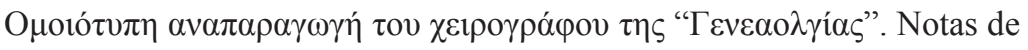
la Genealogía de Kavafis y Reproducción facsimilar del manuscrito de la "Genealogía". Atenas: Archivo Griego Histórico y Literario E$\Lambda I A$.

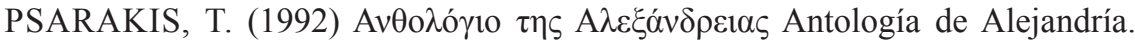
Atenas: Ed. Nea Sínora.

SAVIDI, L. (1983) 'Léfkoma Kavafi 1863-1910, 'Album Kavafis 1863-1910. Atenas: Ed. Hermís.

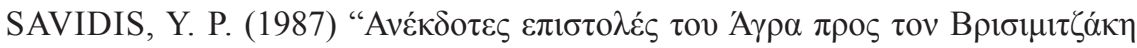

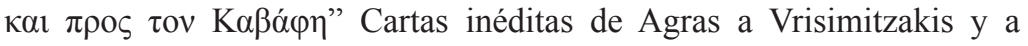

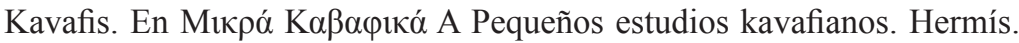
Este artículo contiene el texto de una "larga" carta de Kavafis en respuesta a Agras.

VALIERI-KAVAFI (1992) "O $\theta$ cío

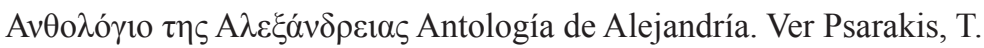

